\title{
School-based educational intervention to improve children's oral health-related behaviors in rural Bangladesh
}

\author{
Yusuke Furukawa ${ }^{1}$, Fumihiko Yokota ${ }^{2}$, Rafiqul Islam Maruf ${ }^{3}$, Mariko Nishikitani $^{4}$, Kimiyo Kikuchi ${ }^{2}$, \\ Ashir Ahmed ${ }^{5}$, Naoki Nakashima ${ }^{6}$
}

${ }^{1}$ Graduate Student, Department of Orthodontics, Kyushu University Hospital, Fukuoka, Japan. ${ }^{2}$ Lecturer; ${ }^{4}$ Associate Professor; Institute of Decision Science for Sustainable Society, Kyushu University, Fukuoka, Japan. ${ }^{3}$ Director, Grameen Communications, Dhaka, Bangladesh. ${ }^{5}$ Associate Professor, Graduate School of Information Science and Electrical Engineering, Kyushu University, Fukuoka, Japan. ${ }^{6}$ Professor, Medical Information Center, Kyushu University Hospital, Fukuoka, Japan.

\begin{abstract}
Children in rural Bangladesh have limited access to oral health services. As a pilot project, a one-day one-time oral health educational intervention was conducted at a primary school in Tangail district in Bangladesh. This study assessed the effect of this educational intervention on the children's oral behaviors using controlled pre- and postintervention design. Fifty-two students at the intervention school and 37 students at a control school agreed to participate in this study. A baseline survey was conducted for children at both schools using a standardized semistructured questionnaire. Children at the intervention school received face-to-face dental exercises and a group seminar. Six months after the intervention, a follow-up survey was conducted at both schools to compare changes in children's oral behaviors at the two schools. The increase in the percentage of intervention-school participants who reported brushing their teeth two or more times per day between the baseline and follow-up surveys was 7.6 times greater than that in the control group $(95 \% \mathrm{CI}=1.7-35.1 ; p<0.009)$. The increase in the percentage of intervention-school participants who reported brushing their teeth before bed between the two surveys was 7.3 times greater than that in the control group $(95 \% \mathrm{CI}=1.6-34.0 ; p<0.001)$. The findings suggest the importance of skill-based intervention, in collaboration with dentists, school teachers, parents, and community health educators. Future studies on the effectiveness of school-based educational interventions should consider longer-term sustainable behavioral changes in children's oral health.
\end{abstract}

Keywords: Health education, Oral health behaviors, School children, Evaluation, Bangladesh.

\section{Introduction}

Oral health has a significant impact on people's general health and quality of life. ${ }^{1-3}$ Previous studies have reported that poor oral health negatively affects the growth, development, and self-esteem of children, as well as their ability to eat, learn, and communicate. ${ }^{1-4}$ Children with poor oral health are 12 times more likely to have restricted days away from school study, including missing school, comparing with those who have better oral health. ${ }^{5}$ Globally each year, oral diseases, particularly dental caries, among children account for more than 50 million lost school hours. ${ }^{6}$ Due to an increasing prevalence of dental caries among schoolchildren in developing countries, ${ }^{3,7-9}$ oral health education to prevent children's dental diseases is urgently needed. ${ }^{10}$

Developing countries continue to be burdened with chronic and infectious diseases, and Bangladeshi children are no exception. Previous reports have suggested that many Bangladeshi children have increased their consumption of snacks and soft drinks, combined with an insufficient use of fluoride and inadequate oral hygiene. ${ }^{4,10}$ In addition, many Bangladeshi children have limited access to oral health services due to financial constraints and a lack of dental care facilities. ${ }^{10}$ Schools therefore are an ideal setting in which to provide oral health education. ${ }^{1}$

\section{Practice Points}

For the prevention of oral diseases, age-specific and skill-based oral health education and dental exercise should be provided for school children in rural Bangladesh.

- School based educational intervention for improving oral health should have collaboration with dentists, school teachers, parents and community leaders.

- There were significantly higher levels of positive behavioral change in the intervention group in brushing their teeth two or more times per day and brushing their teeth before bed at the 6-month follow-up after the school based educational intervention.

- A combined set of oral health exercises and education such as the face-to-face dental practices and oral informational pamphlet could be effective to improve oral health-related behaviors.

- Further investigations are needed to assess the long-term and sustainable behavioral changes in children's oral health.

Correspondence: Fumihiko Yokota, Lecturer, Institute of Decision Science for Sustainable Society, Kyushu University, Fukuoka, Japan. E-mail: yokota.fumihiko.785@m.kyushu-u.ac.jp. 
Although various school-based oral health education programs have been conducted and reported to be successful in many developing and developed countries, ${ }^{2,7,14-16}$ the research on this is limited in a few ways. First, little research on this topic has been conducted in Bangladesh, and the research that has been conducted in Bangladesh has been set in Dhaka City or in areas relatively close to Dhaka city. ${ }^{10-12}$ Thus, the oral health of children in rural areas of Bangladesh is still largely unknown. Second, very few of the previous studies in Bangladesh have examined the effect of a school-based oral health education program focusing on children's oral health behaviors. Improper teethbrushing among school children is the main cause of the development of dental diseases. ${ }^{12}$ One of the studies conducted in Bangladesh reported that only $23 \%$ of secondary school children ages 10 to 13 years were brushing two or more times per day, and only $43 \%$ of them were brushing before bed. ${ }^{13}$ Khan et al. reported that the majority of school children had an adequate level of knowledge on oral health but had a low level of oral health practice. ${ }^{4}$ Thus, research on oral health education should focus more on children's behavioral changes. Finally, although many oral health education interventions have been effective in improving oral health behaviors in developing and developed countries, ${ }^{1,7,14}$ very few previous studies have focused on the effectiveness of age-specific and skill-based education on children's oral health-related behaviors in rural Bangladesh. According to the World Health Organization (WHO), oral health promotion and education needs to focus on developing children's skills to improve and maintain healthy behaviors to reduce the risks of oral diseases. ${ }^{1}$

In consideration of the existing research gap, we aimed in this study to assess the effect of a skill-based educational intervention focusing on fifth-grade children's oral health behaviors at a primary school in Tangail district in Bangladesh.

\section{Materials and methods}

\section{Study design and setting:}

This was a controlled pre- and post-intervention study and was approved by the Kyushu University Institutional Review Board for Clinical Trials (\#24-048). Two primary schools (one for the intervention and the other for the control school) were selected based on their similarities in geography, socioeconomics, and educational programming. Both schools were government primary schools located in the Kalihati subdistrict of Tangail district, which is located approximately $100 \mathrm{~km}$ north-west of Dhaka. According to the 2011 Bangladesh census, the Kalihati sub-district has a population of approximately 400,000 residents. ${ }^{15}$ Community-based health promotion projects have been implemented in the Kalihati sub-district by Grameen groups and Kyushu University in Japan since 2014.

\section{Data collection procedure:}

\section{Baseline survey}

Prior to the baseline survey, a research coordinator from Grameen Communications contacted the principal at the intervention school and the control school, as well as school teachers and community leaders. The coordinator explained the purpose and aims of this study to them. The school principals and teachers contacted their students' parents and community leaders and received a positive response to the study. After receiving an official approval from both school principals and other stakeholders, field research officers and a dental doctor from Grameen Communications and Kyushu University conducted a baseline survey at both schools in August

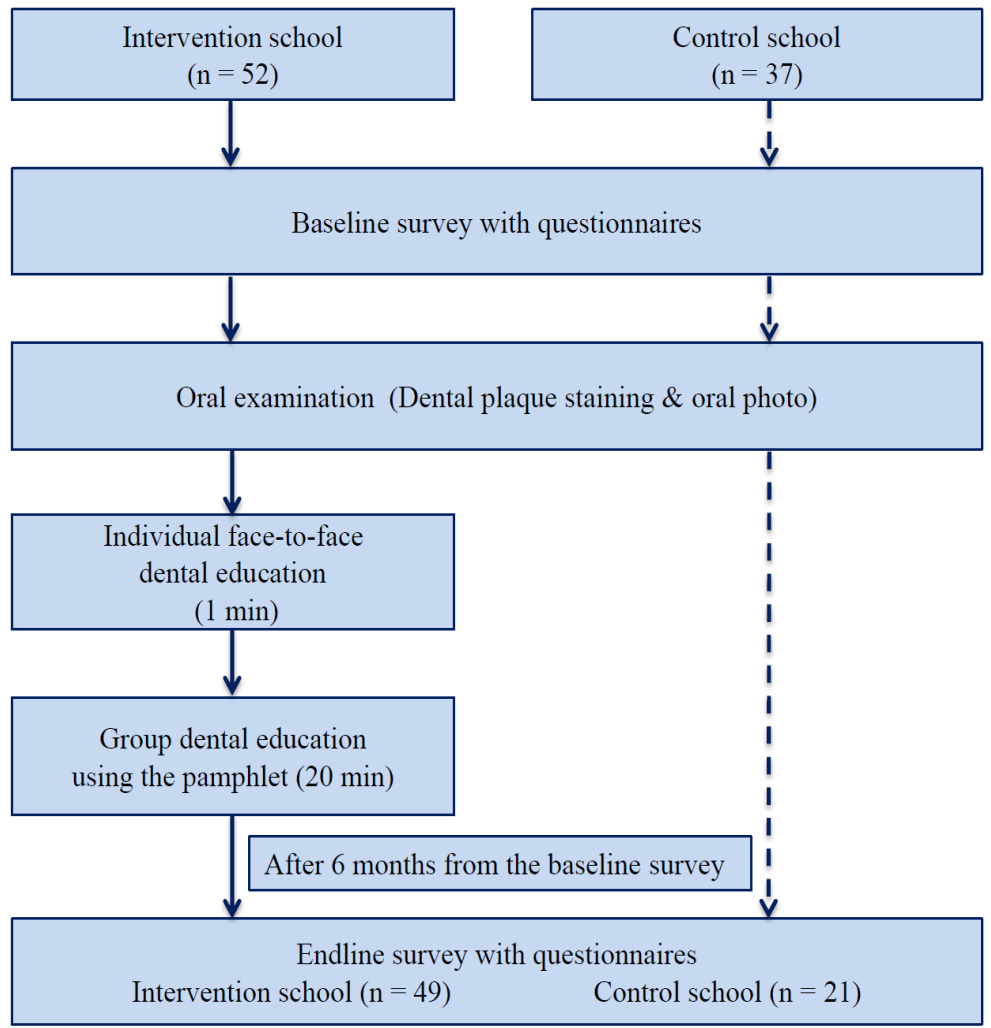

Figure 1. Flow chart of the data-collection process of the study 
2015. A flow chart of the data-collection process in this study is shown in Figure 1. At both the intervention and the control school, all fifth-grade students (52 students from the intervention school and 37 students from the control school) agreed to participate in this study. The baseline survey was conducted using standardized semistructured questionnaires in Bengali, first at the intervention school and the next day at the control school. Before the baseline surveys were given to students, informed consent was obtained verbally from each student. Students were asked to enter the research classroom, where research assistants helped them to answer the questions. The questionnaire includes information on demographic characteristics, such as age, sex, and family background, as well as dentalrelated matters and oral health behavioral information, such as (1) frequency of brushing, (2) practice of brushing before bed, (3) use of commercial tooth brushes and tooth pastes, and (4) frequency of snacking.

Oral examination using dental plaque staining and debris index score

After completion of the baseline survey, all participants from both the intervention school and the control school received an oral examination using dental plaque staining and oral photography by a dentist (Figure 2). First, a dentist stained the dental plaque on the participant's entire tooth surface using a plaque disclosing gel (Herstik Dent Club, Niimi Chemical Industries, Japan). A drop of the staining gel was then placed on a tooth brush, which was applied to the surface of the teeth and rinsed with water. Part of the dental plaque on the surface of the teeth was dyed red. The oral examination was performed in the classroom in natural light. Debris index (DI) scores (range $0-3$, where $0=$ most clean and 3 = least clean) were then calculated based on the oral photos taken.

\section{Intervention 1 (individual face-to-face and group inter-} vention)

After the oral examination, only the intervention school received oral health education, led by a dentist and a health educator from Grameen Communication. First, individual face-to-face instruction on dental exercises was provided by a dentist; the dentist showed the students how to hold the toothbrushes, how to brush, and where to brush. This was followed by a 20 -minute group dental seminar, provided by a health educator in a large classroom to increase awareness of the importance of good dental care. In this session, a 1-page pamphlet with oral health-related illustrations was provided to all

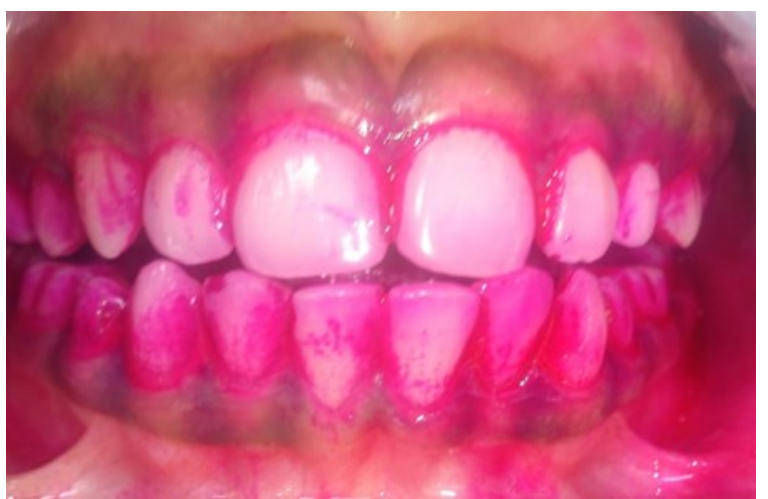

Figure 2: Sample of oral photo taken after staining for dental plaque intervention-group participants. The pamphlet promoted the use of a tooth brush, showed the correct way to hold one, the appropriate amount of toothpaste required, the effectiveness of fluoride toothpastes in the prevention of dental caries, proper brushing methods, and when to brush teeth. The participants could take the pamphlets home, along with tooth brushes and tooth paste samples that were distributed in appreciation.

At the control school, a 20-minute Japanese cultural group session was held by the dentist and health educator. Participants received a sheet of colorful square paper and learned how to make origami.

\section{Follow-up survey}

Six months after the intervention, a follow-up survey was conducted at both schools. At the intervention and control schools, $94 \%$ and $57 \%$ of students respectively who participated in the baseline survey participated in the follow-up survey as well. We had intended to conduct a second oral examination at the follow-up survey, to compare differences in DI scores, but this was not possible due to unavoidable circumstances.

\section{Data analysis}

Person's chi-square test was used to find the distribution and unadjusted difference in baseline characteristics of the study participants at the intervention school and the control school. Fisher's exact test was used when the sample size was smaller than 10. Student's $t$-test was used to compare the DI scores between the two schools. The McNemar test was used to compare the oral health-related behaviors of study participants between the baseline and follow-up surveys at the intervention school and the control school. One question, about 'the participant's communication with family members about their experiences of the oral health education intervention' were asked only in the follow-up survey. This variable was compared between the two schools using chi-square or Fisher's exact test. Multivariable logistic regression analysis was performed to describe age- and sex-adjusted odds ratios to confirm the effect of two behaviors: brushing teeth two or more times per day, and brushing teeth before bed. For this analysis, the interaction terms of school type (intervention school or control school) and survey type (baseline survey or follow-up survey) were included in the model to explain participants' behavioral changes from the baseline survey to the follow-up survey between the intervention school and the control school. All statistical analyses were performed using Stata (version 13, Stata Corp., College Station, TX, USA), and a $p$-value of less than 0.05 was considered to be statistically significant.

\section{Results}

Participant baseline characteristics are shown in Table 1. All 89 students between 10 and 12 years old (52 intervention and 37 control group) agreed to participate in the baseline survey. The age of the participants was significantly different between the two schools $(p=0.004)$. In the control school, more than half of participants $(53 \%)$ were 10 years old, while only $19 \%$ in the intervention group were 10 years old. The primary guardian of the participants 
was also significantly different between the two schools $(p=0.017)$. In the control group, $94 \%$ of participants reported their mother as the primary guardian, compared to $75 \%$ in the intervention group. The mean DI score was significantly higher in the control school $($ mean $=1.61)$ than in the intervention group (mean=1.26) $(p=0.002)$. Other characteristics of the participants, such as sex, number of family members, and experience of oral pain were not significantly different between the two schools. In total, $15 \%$ of participants reported having ever experienced teeth and/or gum pain; however, no participants $(0 \%)$ had ever visited a dental clinic. At the time of the baseline survey, the participants' oral health behaviors of brushing teeth two or more times per day and brushing teeth before bed were not significantly different between the two groups. In total, only $30 \%$ of the participants reported brushing their teeth two or more times per day and only $29 \%$ reported brushing their teeth before bed.

The differences in participants' oral health behaviors between the baseline and follow-up surveys are shown in Table 2. Among participants at the intervention school, the percentage who reported brushing their teeth two or more times per days significantly increased, from 33\% at baseline to $88 \%$ at follow-up $(p<0.001)$, while among participants at the control school, the percentage did not significantly increase. In addition, significant increases were found for many behaviors among the intervention group participants, such as brushing their teeth before bed (31\% at baseline and $88 \%$ at follow-up; $p<0.001$ ), use of a commercial tooth brush $(71 \%$ at baseline and $96 \%$ at follow-up; $p<0.01)$, and use of fluoride tooth paste $(80 \%$ at baseline and $98 \%$ at follow-up; $p<0.001$ ). Conversely, in the control group, there were no significant changes in these behaviors from baseline to follow-up. The percentage of participants who reported having snacks between meals two or more times per day increased significantly in both the intervention group (37\% at baseline and $57 \%$ at follow-up; $p<0.05)$ and the control group (14\% at baseline and $67 \%$ at follow-up; $p<0.01$ ).

The participants' communication with family members about their experiences related to the oral health educational intervention at the follow-up survey are shown in Table 3 . The percentage of participants who had communicated with their grandparents about the oral health educational intervention was higher at the intervention school $(27 \%)$ than at the control school $(5 \%)$. This difference was found to be marginally significant $(p=0.050)$. Communication with other family members, such as fathers, mothers, and brothers/ sisters, did not differ significantly between the two schools at the follow-up survey.

The age- and sex-adjusted odds ratios for the differences between the intervention and control schools in the behavioral outcomes of brushing teeth two or more times per day and brushing teeth before bed are shown in Figure 3. The increase in the percentage of intervention-group participants who reported brushing their teeth two or more times per day between the baseline and follow-up surveys

Table 1: Comparison of baseline characteristics among study participants between the intervention and control schools

\begin{tabular}{|c|c|c|c|c|c|}
\hline \multicolumn{2}{|l|}{ Variables } & \multirow{2}{*}{$\begin{array}{c}\text { Total } \\
\left(\begin{array}{c}\mathbf{N}=\mathbf{8 9}) \\
\text { n (\%) }\end{array}\right. \\
49(55 \%) \\
40(45 \%) \\
\end{array}$} & \multirow{2}{*}{$\begin{array}{c}\begin{array}{c}\text { Intervention school } \\
(\mathbf{N}=\mathbf{5 2}) \\
\mathbf{n}(\mathbf{\%})\end{array} \\
30(58 \%) \\
22(42 \%) \\
\end{array}$} & \multirow{2}{*}{$\begin{array}{c}\text { Control school } \\
\mathbf{n = 3 7} \\
\mathbf{n}(\mathbf{\%}) \\
19(51 \%) \\
18(49 \%) \\
\end{array}$} & \multirow{2}{*}{$\begin{array}{c}\boldsymbol{P}_{\text {-value }}^{\dagger} \\
0.553\end{array}$} \\
\hline Sex & $\begin{array}{l}\text { Male } \\
\text { Female }\end{array}$ & & & & \\
\hline Age*1 $^{* 1}$ & $\begin{array}{l}10 \text { years old } \\
11 \text { years old } \\
12 \text { years old }\end{array}$ & $\begin{array}{l}29(33 \%) \\
49(56 \%) \\
10(11 \%) \\
\end{array}$ & $\begin{array}{c}10(19 \%) \\
34(66 \%) \\
8(15 \%) \\
\end{array}$ & $\begin{array}{c}19(53 \%) \\
15(42 \%) \\
2(5 \%) \\
\end{array}$ & 0.004 \\
\hline $\begin{array}{l}\text { Number of family members in the } \\
\text { household } *^{2}\end{array}$ & $\begin{array}{l}\geq 3 \\
4 \\
\leq 5\end{array}$ & $\begin{array}{c}8(10 \%) \\
19(42 \%) \\
22(49 \%)\end{array}$ & $\begin{array}{c}4(9 \%) \\
19(42 \%) \\
22(49 \%)\end{array}$ & $\begin{array}{l}4(11 \%) \\
21(57 \%) \\
12(32 \%)\end{array}$ & 0.299 \\
\hline Primary guardian $*^{3}$ & $\begin{array}{l}\text { Father } \\
\text { Mother }\end{array}$ & $\begin{array}{l}14(17 \%) \\
67(83 \%)\end{array}$ & $\begin{array}{l}12(27 \%) \\
33(73 \%)\end{array}$ & $\begin{array}{c}2(6 \%) \\
34(94 \%)\end{array}$ & 0.017 \\
\hline Father's occupation $*^{4}$ & $\begin{array}{l}\text { Specialist/Engineer } \\
\text { Business } \\
\text { Expatriate } \\
\text { Farmer } \\
\text { Other }\end{array}$ & $\begin{array}{c}4(5 \%) \\
12(15 \%) \\
13(16 \%) \\
38(48 \%) \\
13(16 \%)\end{array}$ & $\begin{array}{c}2(4 \%) \\
6(14 \%) \\
9(21 \%) \\
17(40 \%) \\
9(21 \%)\end{array}$ & $\begin{array}{c}2(5 \%) \\
6(16 \%) \\
4(11 \%) \\
21(57 \%) \\
4(11 \%)\end{array}$ & 0.434 \\
\hline Mother's occupation*5 & $\begin{array}{l}\text { Housework } \\
\text { Other }\end{array}$ & $\begin{array}{l}63(90 \%) \\
7(10 \%)\end{array}$ & $\begin{array}{c}44(90 \%) \\
5(10 \%)\end{array}$ & $\begin{array}{l}19(90 \%) \\
2(10 \%)\end{array}$ & 1.000 \\
\hline $\begin{array}{l}\text { Have ever experienced teeth and/ } \\
\text { or gum pain? }\end{array}$ & Yes & $18(22 \%)$ & $13(25 \%)$ & $5(14 \%)$ & 0.284 \\
\hline Have ever visited dental clinic? & Yes & 0 & 0 & 0 & N/A \\
\hline $\begin{array}{l}\text { Brushing teeth two or more times } \\
\text { per day? }{ }^{6}\end{array}$ & Yes & $21(30 \%)$ & $16(33 \%)$ & $5(24 \%)$ & 0.459 \\
\hline Brushing teeth before bed? ${ }^{* 6}$ & Yes & $20(29 \%)$ & $15(31 \%)$ & $5(24 \%)$ & 0.564 \\
\hline Using commercial tooth brush?*6 & Yes & $51(73 \%)$ & $35(71 \%)$ & $16(76 \%)$ & 0.681 \\
\hline Using fluoride tooth paste $?^{* 6}$ & Yes & $55(79 \%)$ & $39(80 \%)$ & $16(77 \%)$ & 0.751 \\
\hline $\begin{array}{l}\text { Having snacks between meals two } \\
\text { or more times per day? } *^{6}\end{array}$ & Yes & $21(30 \%)$ & $18(37 \%)$ & $3(14 \%)$ & 0.088 \\
\hline Debris index scores ${ }^{*}$ & Mean $( \pm \mathrm{SD})$ & $1.40( \pm 0.52)$ & $1.26( \pm 0.47)$ & $1.61( \pm 0.52)$ & 0.002 \\
\hline
\end{tabular}

$* \chi^{2}$ test or Fisher's exact test (Student's $t$-test for debris index scores). NA $=$ not applicable. ${ }^{* 1}$ Sample sizes for age for total, intervention group, and control group are 88,52 , and 36 , respectively. ${ }^{2}$ Sample size for "number of family members in the household" for total, intervention group, and control group are 82,45 , and 37 , respectively. $*^{3}$ Sample size for "primary guardian" for total, intervention group, and control group are 81 , 45 , and 36, respectively. ${ }^{* 4}$ Sample size for "father's occupation" for total, intervention group, and control group are 80,43 , and 37 , respectively. $*^{5}$ Sample size for "mother's occupation" for total, intervention group, and control group are 70,49 , and 21 , respectively. ${ }^{6}$ Sample size for total, intervention group, and control group are 70,49 , and 21 , respectively. ${ }^{* 7}$ Sample size for debris index scores for total, intervention group, and control group are 80,47 , and 33 , respectively. 
Table 2: Comparison of participants' oral health behaviors between baseline and follow-up surveys by intervention and control schools

\begin{tabular}{|c|c|c|c|c|c|c|c|}
\hline \multirow[t]{2}{*}{ Variables } & \multirow{2}{*}{$\begin{array}{c}\text { Yes } \\
\text { or } \\
\text { No }\end{array}$} & \multicolumn{3}{|c|}{$\begin{array}{c}\text { Intervention school }(\mathrm{N}=49)^{\mathrm{a}} \\
\text { n }(\%)\end{array}$} & \multicolumn{3}{|c|}{$\begin{array}{c}\text { Control school }(\mathrm{N}=21)^{\mathrm{a}} \\
\text { n }(\%) \\
\end{array}$} \\
\hline & & Baseline & $\begin{array}{l}\text { Follow-up } \\
\text { (6 months) }\end{array}$ & $p$-value & Baseline & $\begin{array}{l}\text { Follow-up } \\
\text { (6 months) }\end{array}$ & $p$-value \\
\hline Brushing teeth two or more times/day & Yes & $16(33 \%)$ & $43(88 \%)^{6 * 6}$ & $<0.001$ & $5(24 \%)$ & $11(52 \%)$ & 0.109 \\
\hline Brushing teeth before bed & Yes & $15(31 \%)$ & $43(88 \%)^{* * *}$ & $<0.001$ & $5(24 \%)$ & $11(52 \%)$ & 0.109 \\
\hline Use of commercial tooth brush & Yes & $35(71 \%)$ & $47(96 \%)$ & 0.002 & $16(79 \%)$ & $21(100 \%)$ & 0.063 \\
\hline Use of fluoride tooth paste & Yes & $39(80 \%)$ & $48(98 \%)$ & 0.012 & $16(79 \%)$ & $21(100 \%)$ & 0.063 \\
\hline Snacks between meals 2 or more times/day & Yes & $18(37 \%)$ & $28(57 \%)^{*}$ & 0.041 & $3(14 \%)$ & $14(67 \%)^{*}$ & 0.001 \\
\hline
\end{tabular}

${ }^{a}$ Students who completed both baseline and follow-up surveys were included. ${ }^{*} p<0.05,{ }^{* *} p<0.01$, and ${ }^{* * *}$ p $<0.001$; comparisons between baseline and follow-up(6 months) within each school were conducted using McNemar's test.

Table 3: Comparison of participants' communication with family members about their experiences related to the oral health educational intervention

\begin{tabular}{|c|c|c|c|c|}
\hline Variables & Family members & $\begin{array}{l}\text { Intervention group } \\
(\mathrm{n}=49)\end{array}$ & $\begin{array}{l}\text { Control group } \\
(n=21)\end{array}$ & $p$-value $\dagger$ \\
\hline $\begin{array}{l}\text { Participants talk about their dental check-up } \\
\text { experiences at school with family members }\end{array}$ & $\begin{array}{l}\text { Father } \\
\text { Mother } \\
\text { Grandparents } \\
\text { Brother/sister } \\
\text { Other relatives }\end{array}$ & $\begin{array}{c}38(78 \%) \\
47(96 \%) \\
13(27 \%) \\
30(61 \%) \\
5(10 \%)\end{array}$ & $\begin{array}{c}16(76 \%) \\
21(100 \%) \\
1(5 \%) \\
8(38 \%) \\
2(10 \%)\end{array}$ & $\begin{array}{l}1.000 \\
1.000 \\
0.050 \\
0.116 \\
1.000\end{array}$ \\
\hline
\end{tabular}

$\chi^{2}$ or Fisher's exact test.

was 7.6 times greater than in the control group (95\% $\mathrm{CI}=1.7-35.1 ; p<0.009)$. The increase in the percentage of intervention-group participants who reported brushing their teeth before sleep between the baseline and follow-up surveys was 7.3 times greater than in the control group $(95 \% \mathrm{CI}=1.6-34.0 ; p<0.001)$ (Figure 3$)$.

\section{Discussion}

To our knowledge, this is the first study to investigate the effect of a school-based educational intervention on children's oral health-related behaviors in rural Bangladesh. In this small-scale, one-time intervention, the key finding was that there were significantly higher levels of positive behavioral change in the intervention group in brushing their teeth two or more times per day and brushing their teeth before bed at the 6-month follow-up.

Consistent with most previous studies, the results of this

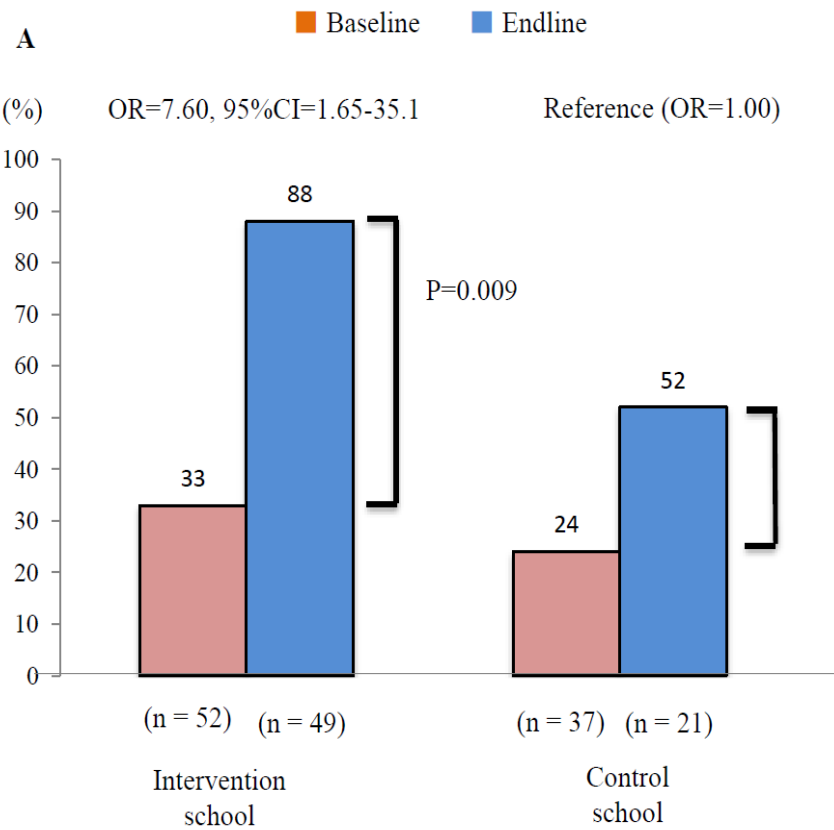

study suggest that school-based oral health education may have positive effects on brushing practices among school children. ${ }^{7,14,15}$ One possible reason for this may be that the skill based face-to-face dental exercises conducted by a dentist were well accepted by the participating school children. The exercises focused on improving fifth-grade children's teeth-brushing skills. Since most children have 28 of their permanent teeth by age 13 years, ${ }^{18}$ fifth grade is a critical time to develop oral health habits, like proper methods for holding a tooth brush and how and when to brush. Such agespecific and skill-based approaches are also recommended for oral health education by the WHO. ${ }^{1}$ Furthermore, the group educational seminar might have been effective in encouraging positive oral behavioral change among the intervention-school children. Also in the seminar, pamphlets with important oral healthrelated information were used and taken home. More than $78 \%$ of the intervention-school children reported
Baseline $\quad$ Endline

B

(\%) $\mathrm{OR}=7.30,95 \% \mathrm{CI}=1.57-34.0 \quad$ Reference $(\mathrm{OR}=1.00)$

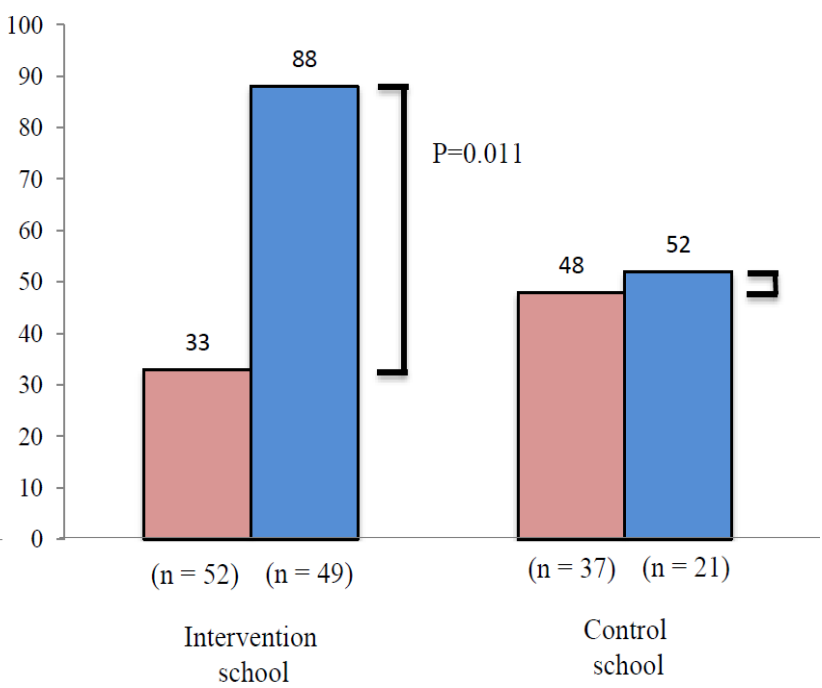

Figure 3: Participants' behavior regarding oral health: (A) brushing teeth two or more times per day and (B) brushing teeth before bed. 
that they talked with their father or mother about their experiences in the oral health exercise and seminar. In addition, $27 \%$ of them talked with their grandparents, compared with only $5 \%$ of the children at the control school. This finding indicates that children's communication with family members may have a positive influence on children's oral health-related behaviors.

Another potential reason for positive effects of the intervention may be due to wide range of community acceptance for our educational. ${ }^{1}$ intervention. Although our educational intervention was simple and took very little time, there was much support from and collaboration with multiple stakeholders, including school principals, teachers, parents, community leaders, and researchers at Grameen Communications. Such a supportive environment is important in a school-based oral health promotion Previous studies have also reported that parental/guardian support is crucial in the development of healthy habits in children. ${ }^{17}$ Although this educational intervention was only one day and onetime, field research staff spent a lot of time with all stakeholders in preparation and follow-up before and after the intervention.

The study has some limitations. First, the study intervention sample from a single primary school is unlikely to be representative of all fifth-grade school children in Tangail district. The study results are limited to the fifth-grade children who voluntarily participated in the oral health educational intervention in Tangail district. However, this study could potentially be a model case for interventions to improve oral healthrelated behaviors in children of a similar age in rural Bangladesh who have never visited a dentist. Second, our analysis for the effect of interventional oral hygiene education evaluated only subjective changes, such as self-reported oral health behaviors. The follow-up survey did not allow us to measure objective indicators, such as DI scores, due to security constraints resulting from concerns about terrorism. Further studies should include objective measurements associated with children's oral health-related behaviors. Third, although much time was spent on preparation and follow-up before and after the intervention, the actual intervention took place only once, on a single day. This is because this study was conducted as a pilot project of a schoolbased oral health education model in rural Bangladesh in order to improve and maintain good oral health. This one-day and one-time approach may produce only short -term behavioral changes but may not be sustain good oral health habits. It will be more sustainable if the intervention could be integrated into the regular school curriculum in the future.

\section{Conclusion}

Despite these limitations, this study found positive effects resulting from the school-based educational intervention on rural Bangladeshi children's oral healthrelated behaviors: brushing teeth two or more times per day and brushing teeth before bed. The finding suggests the importance of age-specific and skill-based intervention in collaboration with dentists, school teachers, parents, and community leaders, even if the intervention is only one day and one time. It also indicated that the face-to-face dental practices let by a dentist and proper contents of the oral informational pamphlet were effective in helping to improve children's oral health-related behaviors 6 months after the intervention. Future studies on the effectiveness of school-based educational interventions should consider more long-term and sustainable behavioral changes in children's oral health.

\section{Competing interest}

The authors declare that they have no competing interests.

\section{Competing interest}

This research project described in this paper was supported by Global Communication Center of Grameen Communications in Bangladesh. We would like to thank all the students, as well as their parents, teachers, and principals, for their cooperation in our intervention study.

\section{References}

1. World Health Organization. Oral health promotion: an essential element of a healthpromoting school. WHO Information Series on School Health. Document11. Geneva: WHO, 2003

2. Kwan SYL, Petersen PE, Pine CM, Borutta A. Health-promoting schools: an opportunity for oral health promotion. Bull World Health Organ 2005;83:677-85.

3. Petersen PE. Sociobehavioral risk factors in dental caries; international perspective. Соттиnity Dent Oral Epidemiol 2005;33:274-9.

4. Khan MHA, Zaman K, Islam S, Islam MM, Sarwar MG, Rahman MM. Evaluation of school oral health education program; A review. Bangladesh J Dent Res Educ 2013;3(2):45-50.

5. US General Accounting Offices. Oral Health: Dental Disease is a Chronic Problem among Low Income Populations. Report to Congressional Requesters. Washington; 2000.

6. Gift HC, Reisine ST, Larach DC. The social impact of dental problems and visits. $\mathrm{Am} \mathrm{J}$ Public Health 1992;82:1663-8.

7. Jurgensen N. Petersen PE. Promoting oral health of children through schools; Results from a WHO global survey 2012. Community Dent Health 2013;30(4):204-18.

8. David J, Wang NJ, Astrøm A.N, Kuriakose S. Dental caries and associated factors in 12-yearold schoolchildren in Thiruvananthapuram, Kerala, India. Int J Paediatr Dent 2005;15:420-8.

9. Petersen PE, Hoerup N, Poomviset N, Prommajan J, Watanapa A. Oral health status and oral health behavior of urban and rural schoolchildren in Southern Thailand. Int Dent J 2001;51:95-102.

10. Haque SE, Rahman M, Kawashima I, Mutahara M, Sakisaka K, Tsutsumi A, Islam MJ, Mostofa MG. Effect of a school-based oral health education in preventing untreated dental caries and increasing knowledge, attitude, and practices 
among adolescents in Bangladesh. BMC Oral Health 2016;16:44.

11. Farzan MSA, Zayeed MA, Debnath S, Zerin I, Afrin S. Oral health condition among selected school children in Dhaka city. Bangladesh J Dent Res \& Edu 2013;3(1):21-4.

12. Fakir MM, Alam KMU, Mamun FA, Sarker N. A survey on oral health condition in primary school children. Medicine today 2010;22(2):701.

13. Hayet SMA, Islam MH, Awal MA. Knowledge on oral hygiene and oral health status among the secondary school students. Int $J$ Dent Med 2015;1(2):17-21.

14. Nakre PD, Harikiran AG. Effectiveness of oral health education programs: A systematic review. J Int Soc Prev \& Com Dent 2013;3(2):103-15.
15. Kesavan R. Mary AV. Effectiveness of dental health education. A systematic review. $J$ Ind Assoc Pub Health Dent 2011;18(1):345-50.

16. Gambhir RS, Sohi RK, Nanda T, Sawhney GS, Setia S. Impact of school based oral health education programmes in India: A systematic review. J of Clin and Diagn Res 2013;7(12):3107 -10 .

17. Petersen PE, Peng B, Tai B, Bian Z, Fan M. Effect of a school-based oral health education programme in Wuhan City, Peoples Republic of China. Int Dent J 2004;54(1):33-41.

18. ADA Division of Communications. Tooth eruption: The permanent teeth. J of Am Dent Assoc 2006;137(1):127. 